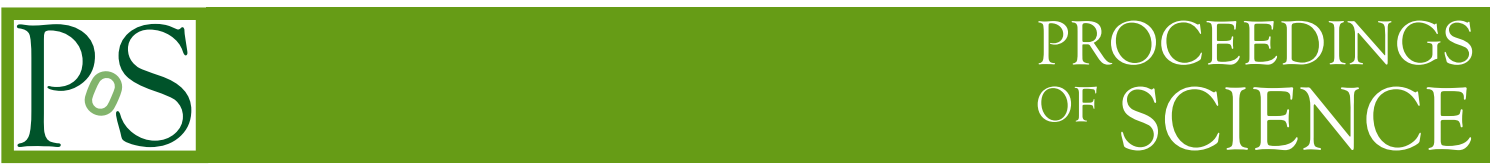

\title{
GX 301-2 with INTEGRAL
}

\author{
V. Doroshenko*1, R. Staubert ${ }^{1}$, I. Kreykenbohm ${ }^{2,4}$, A. Santangelo ${ }^{1}$ and C. Ferrigno ${ }^{1,3}$ \\ ${ }^{1}$ Institut für Astronomie und Astrophysik, Sand 1, 72076 Tübingen, Germany \\ ${ }^{2}$ Dr. Karl Remeis-Sternwarte, Sternwartstrasse 7, 96049 Bamberg, Germany \\ ${ }^{3}$ ISDC Data Centre for Astrophysics, Chemin d'Écogia 16, CH-1290 Versoix, Switzerland \\ ${ }^{4}$ Erlangen Centre for Astroparticle Physics (ECAP), Erwin-Rommel-Strasse 1, 91058 Erlangen, \\ Germany \\ E-mail: \\ V.Doroshenko@astro.uni-tuebingen.de, \\ Ingo.Kreykenbohmesternwarte.uni-erlangen.de, \\ Andrea.Santangelo@uni-tuebingen.de, Carlo.Ferrigno@unige.ch
}

We present the results of an in depth analysis of the High Mass X-ray Binary GX 301-2 using INTEGRAL data. The source has a double-peaked orbital lightcurve with flares close to orbital phases 0.5 and 0.9 . In addition to archival data three dedicated observations with a total exposure time of $200 \mathrm{ksec}$ were performed to observe the pre-periastron flare of the source when the flux from the source is maximal. We obtained broad-band $(2-100 \mathrm{keV})$ pulse phase resolved spectra. We discuss the spectral models suitable to describe the spectrum and the impact of the model on the dependence of the cyclotron resonance scattering feature (CRSF) energy with pulse phase. The history of the pulse period since the launch of INTEGRAL is determined and the spin down trend, established by RXTE [1, 2], and BeppoSAX [3] observations is confirmed. Additionally we provide an updated orbital period value based on time arrival analysis of archival data which is found to be consistent with the period obtained from the RXTE ASM lightcurve of the source.

7th INTEGRAL Workshop

September 8-11 2008

Copenhagen, Denmark

\footnotetext{
* Speaker.
} 


\section{Introduction}

GX 301-2 (also known as 4U 1223-62) is a High Mass X-ray Binary system, consisting of a neutron star orbiting the B-type optical companion Wray 977 . The neutron star is a $\sim 680 \mathrm{~s}$ X-ray pulsar [4], accreting from the dense wind of the optical companion. The wind mass loss rate of the optical component is one of the highest known in the galaxy $\left(\dot{M} \sim 10^{-5} M_{\odot} \mathrm{yr}^{-1}\right)$. As the terminal velocity of the wind is very low $\left(v_{0} \sim 300 \mathrm{~km} \mathrm{~s}^{-1}\right.$, [5]), the accretion rate is high enough to explain the observed luminosity of the order of $L_{\mathrm{X}} \sim 10^{37} \mathrm{ergs}^{-1}$. The distance to the source is estimated between $1.8 \mathrm{kpc}$ [6] and $5.3 \mathrm{kpc}$ [7] depending on the spectral classification of Wray 977, with the latest estimate close to $3 \mathrm{kpc}$ [5]. We will adopt the latter value throughout the paper. The source exhibits regular X-ray flares about $1-2 \mathrm{~d}$ before periastron passage (orbital phase $\sim 0.9$ ). There is also an indication for a second flare at orbital phase $\sim 0.5$ [8]. Several hypotheses were proposed to explain the observed orbital lightcurve, including a circumstellar disk [8] as well as the presence of a quasi-stable accretion stream [9]. In this paper we focus mainly on studying the properties of the source during the pre-periastron flare, where the source is brightest, such that the spectral parameters can be obtained with very high significance. The spectrum of the source is complex: modelling the low energy part requires the usage of partial covering models with an optional reflection component. An iron line with a complicated shape is observed at $\sim 6.5 \mathrm{keV}[10]$. A high energy cutoff at $\sim 20 \mathrm{keV}$ together with a deep and broad cyclotron resonance scattering feature (CRSF) at $\sim 30-45 \mathrm{keV}$ are present at higher energies.

\section{Observations and data selection}

The International Gamma-Ray Astronomy Laboratory (INTEGRAL) launched in October, 2002 by the European Space Agency is equipped with 3 co-aligned coded mask instruments: IBIS (Imager on board INTEGRAL Satellite, [11] ), JEM-X (Joint European X-ray monitor, [12]), and SPI (Spectrometer on INTEGRAL, [13]). In this paper, however, we rely on data from IBIS (ISGRI layer) and JEM-X only. A set of pointed observations was performed to observe the source with INTEGRAL during orbital phase $\sim 0.9$ to study the source during the pre-periastron flare. The parameters of the 3 observations are given in Table 2 .

Table 1: Pointed observations of GX 301-2 by INTEGRAL. The updated orbital period value is used to calculate orbital phase and determine the pulse period.

\begin{tabular}{|c|c|c|c|c|c|}
\hline $\begin{array}{c}\text { INTEGRAL } \\
\text { science window }\end{array}$ & $\begin{array}{c}\text { MJD of } \\
\text { observation }\end{array}$ & $\begin{array}{l}\text { Orbital } \\
\text { phase }\end{array}$ & $\begin{array}{l}\text { exposure, } \\
\text { ksec }\end{array}$ & $\begin{array}{c}\text { rate, } \\
\text { ISGRI }_{20-60 \mathrm{keV}} \mathrm{cts} \mathrm{s}^{-1}\end{array}$ & $\begin{array}{c}\text { Pulse } \\
\text { period, s. }\end{array}$ \\
\hline 05180027-66 & $54110.52-12.22$ & $0.96-1.02$ & 91.66 & 98 & $684.37 \pm 0.01$ \\
\hline 05730048-60 & $54276.28-76.85$ & $0.95-0.97$ & 31.98 & 163 & $685.12 \pm 0.01$ \\
\hline $05740012-40$ & $54277.63-78.91$ & $0.99-1.02$ & 69.38 & 76 & $685.17 \pm 0.07$ \\
\hline
\end{tabular}

To study the timing properties of the source in addition to our pointed observations we used IBIS archival data. We analyzed all data when GX 301-2 was within the half coded field of view 
Table 2: Period values obtained using INTEGRAL data. The updated orbital period value was used to correct the lightcurve for the binary motion. The last 5 entries correspond to our pointed observations. Precise period values for the 3 pointed observations performed are listed in Table 1.

\begin{tabular}{cccccccc}
\hline$M J D_{\text {obs }}$ & $P_{\text {spin }}, \mathrm{s}$. & $M J D_{\text {obs }}$ & $P_{\text {spin }}, \mathrm{s}$. & $M J D_{\text {obs }}$ & $P_{\text {spin }}, \mathrm{s}$. & $M J D_{\text {obs }}$ & $P_{\text {spin }}, \mathrm{s}$. \\
\hline 52789 & $681.0 \pm 0.3$ & 53537 & $684.8 \pm 0.3$ & 53526 & $683.9 \pm 0.1$ & 53548 & $684.4 \pm 0.3$ \\
52790 & $681.5 \pm 0.3$ & 53539 & $683.8 \pm 0.3$ & 53527 & $684.0 \pm 0.3$ & 53549 & $684.1 \pm 0.1$ \\
52791 & $681.4 \pm 0.2$ & 53540 & $684.3 \pm 0.4$ & 53528 & $683.4 \pm 0.5$ & 54108 & $684.6 \pm 0.4$ \\
52796 & $680.8 \pm 0.5$ & 53542 & $684.2 \pm 0.4$ & 53529 & $684.2 \pm 0.1$ & 54110 & $684.6 \pm 0.2$ \\
53086 & $683.6 \pm 0.1$ & 53544 & $684.4 \pm 1.0$ & 53533 & $684.9 \pm 0.2$ & 54112 & $684.7 \pm 0.1$ \\
53088 & $683.5 \pm 0.1$ & 53545 & $684.3 \pm 0.1$ & 53534 & $684.6 \pm 0.3$ & 54276 & $685.4 \pm 0.1$ \\
53524 & $683.3 \pm 0.3$ & 53546 & $684.7 \pm 0.4$ & 53536 & $685.5 \pm 0.4$ & 54278 & $685.7 \pm 0.1$ \\
53525 & $683.9 \pm 0.3$ & 53547 & $684.1 \pm 0.5$ & & & & \\
\hline
\end{tabular}

of IBIS (554 science windows in total within $9^{\circ}$ from the center) to obtain the source lightcurve. To determine the orbital parameters, we used the longest almost continuous INTEGRAL observations (revolutions 322-330, $283 \mathrm{scw}$ in total) of the source, covering almost a complete binary cycle.

\section{Data analysis}

\subsection{Timing analysis}

For the timing analysis we used all available IBIS data where the source was within $9^{\circ}$ from the center of the FOV. We determined the pulse period using epoch folding for a set of semicontinuous parts of the source lightcurve. We used $10 \%$ of the $\chi^{2}$ peak width in the periodogram as an estimate for the error. The obtained values for the pulse period are shown in Table 3.1. It is apparent that the spin down trend established by BeppoSAX and RXTE observations continues. A quadratic fit to the INTEGRAL data was performed to obtain qualitative estimates for this trend: $P \sim 681.4 \mathrm{~s}, \dot{P} \sim 6 \times 10^{-8} \mathrm{~s} \mathrm{~s}^{-1}, \ddot{P} \sim-2 \times 10^{-11} \mathrm{~s} \mathrm{~s}^{-2}$.

To study timing properties on the shorter timescales it is no longer possible to use epoch folding, instead the pulse phase connection method is used. A fixed phase of a periodic signal is observed at times

$$
t_{n}=t_{0}+P_{0} n+\frac{1}{2} \dot{P} P_{0} n^{2}+\frac{1}{6} \ddot{P} P_{0}^{2} n^{3}+a \sin (i) F_{n}(e, \omega, \tau, \theta)
$$

referred to as time of arrival (TOA) below, where $P_{0}$ is the pulse period at the initial epoch $t_{0}$, and $\dot{P}, \ddot{P} \ldots$ is the time derivatives of $P_{0}$. The last term of Eq. 3.1 represents the Doppler delays caused by the effects of the orbital motion as a function of the Kepler parameters for an eccentric orbit: the projected semi-major axis $a \sin (i)$ in light seconds ( $i$ is the orbit inclination), the eccentricity $e$, the longitude of the periastron $\omega$, time of periastron passage $\tau$, and the mean anomaly $\theta=$ $2 \pi(t-\tau) / P_{\text {orb }}$. To obtain a solution for the unknown pulse and orbital parameters, a number of measurements of $t_{n}$ for each known $n$ must be obtained. Usually only $t_{\mathrm{n}, \mathrm{obs}}$ is measured, while $n$ must be found during the fitting procedure to obtain a self consistent solution. 
Unfortunately span of even longest observation did not allow us to fit for all of the orbital parameters. We fixed all the parameters except pulse period, its derivative and periastron passage time of the current cycle to published values. The best fit values are $P=684.1618(2) \mathrm{s}, \dot{P}=$ $4.25(2) \times 10^{-8} \mathrm{~s} \mathrm{~s}^{-1}$ and $T_{\mathrm{PA}}=53531.63(1)$ (all uncertainties are at $1 \sigma$ confidence level). Orbital period may be calculated by comparing our $T_{\mathrm{PA}}$ value with that reported by [8] and assuming constant period. It turns out to be significantly less than reported earlier $P_{\text {orb }}=41.482(2)$, which makes the assumption of a constant period questionable. Our estimate for secular change of orbital period using $T_{\mathrm{PA}}$ values by $[14,8]$ is $\sim-3.6 \times 10^{-6} \mathrm{dd}^{-1}$.

We checked this also by measuring phases of the pre-periastron flares associated with the orbital motion. Applying the technique described above to the RXTE-ASM data of GX 301-2 we obtained a value of $P_{\text {orb }}=41.482(4)$ consistent with the $P_{\text {orb }}$ obtained above for constant period case with timing measurements. Although it is not possible to see any period variations within ASM data, the period derivative obtained by fixing orbital period value at time reported by [14] values is consistent with estimate above $\left(\sim-3.2 \times 10^{-6} \mathrm{dd}^{-1}\right)$.

\subsection{Spectral analysis}

Due to the lack of high quality theoretical models, spectra of X-ray pulsars are most often described with phenomenological multi-component models. Typically used components include a power law, a high energy cutoff, and photoelectric absorption. Sometimes a Gaussian absorption line is introduced to account for the CRSF.

$$
I_{\mathrm{NS}}(E)=I_{\mathrm{abs}}(E) \times\left(A_{\Gamma} \times E^{-\Gamma} \times I_{\text {cutoff }} \times \exp \left(-\tau_{\mathrm{gabs}}(E)\right)+I_{\mathrm{Fe}}(E)\right)
$$

Here $A_{\Gamma}$ is a normalization constant, $I_{\mathrm{Fe}}$ an optional iron line, $I_{\mathrm{abs}}$ accounts for the photoelectric absorption, and the last component models a CRSF with a Gaussian shaped optical depth profile [15]. An iron emission line $I_{\mathrm{Fe}}(E)$ is usually treated as a simple Gaussian line.

$I_{\text {abs }}$ accounts for the photoelectric absorption at lower energies. In a clumpy wind accreting system we can expect that a part of the X-rays from the neutron star is subject to strong photoelectric absorption. All radiation emerging from the emission region is also subject to a different amount of absorption. The resulting spectrum is a superposition of several different absorption column densities. It is clear that it is almost impossible to separate contributions from individual absorption columns such that only a small number of photoelectric absorption components is required. For example [2] and [3] adopted two columns to describe their spectra. We therefore also use only two column densities effectively, but fix one to zero (pcfabs model in XSPEC):

$$
I_{\mathrm{abs}}=f \exp \left[-N_{\mathrm{H}} \sigma(E)\right]+(1-f)
$$

where $N_{\mathrm{H}}$ is the equivalent hydrogen column and $\mathrm{f}$ is the covering fraction $(0<f<1)$. For $I_{\text {cutoff }}$ [2] used the Fermi-Dirac cutoff [16]

$$
I_{\text {cutoff }}=\frac{1}{\exp \left(\left(E-E_{\text {cut }}\right) / E_{\text {fold }}+1\right)}
$$


Table 3: Fit results for phase averaged spectra. See text for discussion of the models and the parameters.

\begin{tabular}{|c|c|c|c|c|}
\hline Parameter & Fermi-Dirac & High-energy cutoff & & Becker\& Wolf(2007) \\
\hline$n_{\mathrm{H}}$ & $124.2_{-17.3}^{+10.4}$ & $153.0_{-1.0}^{+-1.0}$ & & $219.3_{-19.1}^{+18.5}$ \\
\hline$f$ & $0.53_{-0.01}^{+0.0163}$ & $0.74_{-12.14}^{+11.03}$ & & $0.56_{-0.1}^{+0.07}$ \\
\hline$E_{\mathrm{cyc}}$ & $39.72_{-1.26}^{+2.74}$ & $46.13_{-0.08}^{+-0.03}$ & & $40.6_{-2.8}^{+1.4}$ \\
\hline$\sigma_{\mathrm{cyc}}$ & $11.68_{-1.04}^{+1.03}$ & $10.9_{-1.0}^{+1.7}$ & & $14.2_{-2.6}^{+2.7}$ \\
\hline$\tau_{\mathrm{cyc}}$ & $24.04_{-8.48}^{+19.02}$ & $25.6_{-1.3}^{+1.4}$ & & $59.1_{-28.3}^{+42.0}$ \\
\hline$\Gamma$ & $-0.61_{-0.12}^{+0.15}$ & $-0.2_{-6.2}^{+11.4}$ & & - \\
\hline$A_{\Gamma}$ & $0.020_{-0.005}^{+0.007}$ & $0.03_{-0.09}^{+0.07}$ & $\xi$ & $0.42_{-0.08}^{+0.13}$ \\
\hline$E_{\text {cut }}$ & $13.15_{-1.34}^{+1.88}$ & $13.611_{-0.007}^{+-0.002}$ & $\delta$ & $0.98_{-0.5}^{+0.08}$ \\
\hline$E_{\text {fold }}$ & $5.2_{-0.1}^{+0.4}$ & $6.6_{--0.3}^{+1.6}$ & $T_{e}$ & $4.1_{-0.4}^{+0.5}$ \\
\hline$\sigma_{\text {fold }}$ & - & $15.5_{-0.4}^{+0.4}$ & $r_{0}$ & $17.5_{-0.5}^{+5.5}$ \\
\hline$E_{\mathrm{Fe}}$ & $6.35_{-0.04}^{+0.04}$ & $6.3_{-3.4}^{+4.0}$ & & $6.26_{-0.03}^{+0.04}$ \\
\hline$\sigma_{\mathrm{Fe}}$ & $0.46_{-0.05}^{+0.04}$ & $0.39_{-0.01}^{+0.03}$ & & $0.55_{-0.07}^{+0.05}$ \\
\hline$A_{\mathrm{Fe}}$ & $0.020_{-0.001}^{+0.002}$ & $0.01_{-0.04}^{+0.05}$ & & $0.026_{-0.002}^{+0.002}$ \\
\hline$\chi^{2} / d o f$ & $1.17 / 146$ & $1.33 / 147$ & & $1.18 / 147$ \\
\hline
\end{tabular}

where $E_{\text {cut }}$ and $E_{\text {fold }}$ are called cutoff and folding energy of the Fermi-Dirac cutoff, respectively. [3] used a different model to describe the cutoff. It is based on the highecut model in XSPEC

$$
I_{\text {cutoff }}= \begin{cases}\exp \left(\left(E_{\text {cut }}-E\right) / E_{\text {fold }}\right) & E \geq E_{\text {cut }} \\ 1 & E \leq E_{\text {cut }}\end{cases}
$$

but it was modified in a way that the transition region is smoothed to avoid cusps.

In addition we use a theoretical model developed by [17]; we refer to the original paper for a detailed discussion of this model and its parameters. The model is based on a detailed treatment of both bulk and thermal Comptonization of a seed radiation occurring in the accretion columns of X-ray pulsars. Taking all constraints into account the following parameters are used for fitting the data: the column radius $r_{0}$, the temperature of the electrons in the optically thin region above the thermal mound $T_{e}$, the magnetic field strength $\mathrm{B}$, the accretion rate $\dot{M}$, and the dimensionless parameters $\xi$ and $\delta$ where $\delta$ accounts for a relative contribution from the thermal and bulk comptonizations, while $\xi$ determines the importance of the escape of photons from the accretion column.

Using the standard OSA 6.1 pipeline we obtained a phase averaged spectrum. In a first step we fitted it with the models described above. The fit results are shown in Table 3.2; the unfolded spectrum and the residuals for fits with and without the inclusion of a CRSF are shown in Fig. 1.

In a next step we performed pulse phase resolved analysis using the data from revolutions 518 and 573 as no evolution of the pulse profile is evident during these revolutions. The corresponding fit results for the Fermi-Dirac cutoff model are shown in Table 3.2. Most resulting parameters are consistent with the values reported by [2]. 

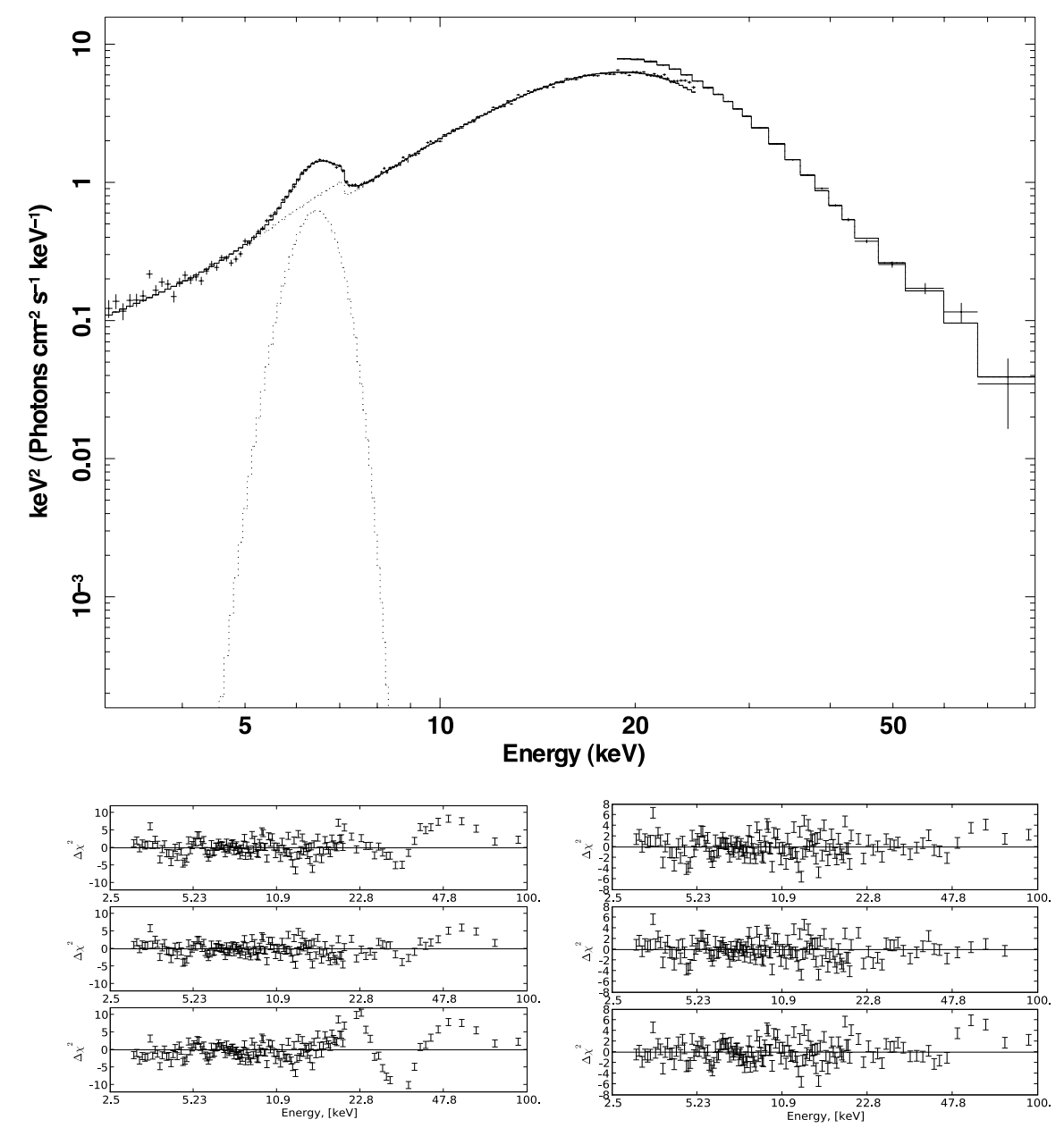

Figure 1: Unfolded spectrum and residuals for the Fermi-Dirac, the high energy cutoff, and the Becker-Wolf models (top to bottom), with (right) and without (left) inclusion of a CRSF.

The self consistency of the model by [17], however, must be checked. The bolometric luminosity can be estimated from the accretion rate,

$$
L_{X}=\frac{G M_{\mathrm{NS}} \dot{M}}{R_{\mathrm{NS}}} .
$$

As $\dot{M}$ is one of model parameters, it is possible to check if the model flux is consistent with the observed flux. The model does not include any angular dependence, therefore it only applies to the phase averaged spectra. The distance to the source was fixed at $3 \mathrm{kpc}$ and the neutron star mass at $1.85 M_{\odot}$ [5]. To make the model flux and the estimate from $\dot{M}, D$ compatible, $\dot{M}$ has to be of the order of $\sim 0.810^{17} \mathrm{gs}^{-1}$. The magnetic field strength can be obtained from the energy of the CRSF:

$$
B_{12}=11.57 \frac{1}{1+z} E_{\mathrm{cyc}}
$$

where $z=\frac{1}{\sqrt{1-\frac{2 G M}{R c^{2}}}}-1$ is the gravitational red shift and $B_{12}$ is the magnetic field strength in units of $10^{12} \mathrm{G}$. The fit results of the variable parameters are shown in Table 3.2. These parameters 
Table 4: Spectral fit results of the phase resolved analysis results for the Fermi-Dirac cutoff model. All uncertainties are on a $3 \sigma$ level. See text for a discussion of the model.

\begin{tabular}{lccccc}
\hline Par./Phase & $0-0.2$ & $0.2-0.4$ & $0.4-0.6$ & $0.6-0.8$ & $0.8-1.0$ \\
\hline$E_{\text {cyc }}[\mathrm{keV}]$ & $29.7_{-1.1}^{+1.0}$ & $38.1_{-1.3}^{+2.3}$ & $36.8_{-1.3}^{+1.3}$ & $34.4_{-1.0}^{+0.4}$ & $29.9_{-3.2}^{+7.4}$ \\
$\sigma_{\text {cyc }}[\mathrm{keV}]$ & $3.7_{-2.1}^{+19.1}$ & $7.7_{-1.6}^{+2.2}$ & $6.7_{-1.5}^{+1.7}$ & $7.8_{-2.7}^{+3.4}$ & $2.7_{-1.5}^{+3.5}$ \\
$\tau_{\text {cyc }}$ & $1.0_{-0.4}^{+0.7}$ & $9.7_{-0.4}^{+0.7}$ & $8.6_{-2.8}^{+2.4}$ & $8.9_{-3.8}^{+3.3}$ & $0.8_{-0.6}^{+3.4}$ \\
$N_{H}\left[10^{22}\right]$ & $187.5_{-28.0}^{+26.2}$ & $116.8_{-23.38}^{+26.5}$ & $118.1_{-35.6}^{+39.2}$ & $71.6_{-7.4}^{+10.5}$ & $101.0_{-33.6}^{+101.2}$ \\
$f$ & $0.57_{-0.07}^{+0.07}$ & $0.54_{-0.03}^{+0.05}$ & $0.45_{-0.04}^{+0.07}$ & $0.71_{-0.03}^{+0.03}$ & $0.53_{-0.08}^{+0.16}$ \\
$\Gamma$ & $-0.9_{-0.2}^{+0.2}$ & $-0.9_{-0.3}^{+0.1}$ & $-0.9_{-0.2}^{+0.2}$ & $-0.17_{-0.21}^{+0.08}$ & $-0.8_{-0.5}^{+0.4}$ \\
$E_{\text {cut }}[\mathrm{keV}]$ & $11.7_{-1.9}^{+1.7}$ & $11.4_{-1.9}^{+1.6}$ & $12.7_{-1.5}^{+2.0}$ & $18.1_{-0.5}^{+2.4}$ & $14.7_{-4.5}^{+1.9}$ \\
$E_{\mathrm{fold}}[\mathrm{keV}]$ & $4.48_{-0.11}^{+0.07}$ & $5.11_{-0.13}^{+0.09}$ & $4.7_{-0.2}^{+0.2}$ & $4.87_{-0.15}^{+0.05}$ & $4.6_{-0.1}^{+0.2}$ \\
$E_{\mathrm{Fe}}[\mathrm{keV}]$ & $6.32_{-0.05}^{+0.05}$ & $6.32_{-0.05}^{+0.05}$ & $6.33_{-0.05}^{+0.05}$ & $6.35_{-0.06}^{+0.06}$ & $6.34_{-0.05}^{+0.05}$ \\
$\sigma_{\mathrm{Fe}}[\mathrm{keV}]$ & $0.44_{-0.09}^{+0.05}$ & $0.47_{-0.09}^{+0.05}$ & $0.58_{-0.08}^{+0.1}$ & $0.38_{-0.11}^{+0.1}$ & $0.44_{-0.09}^{+0.1}$ \\
$\chi^{2} / \mathrm{dof}$ & $0.92 / 41$ & $1.58 / 44$ & $1.0 / 43$ & $1.19 / 45$ & $1.15 / 46$ \\
\hline$\chi_{\mathrm{red}}^{2}$ & 1.13 & 1.51 & 1.36 & 1.28 & 1.30 \\
\hline
\end{tabular}

describe the GX 301-2 spectrum well in a self-consistent way and satisfy all known observational constraints. These parameters imply that the spectrum is dominated by a thermally Comptonized bremsstrahlung emission spectrum.

\section{Conclusions}

Using archival INTEGRAL data GX 301-2 we determined the evolution of the pulse period and a spin-down of the order of $\sim 2 \mathrm{syr}^{-1}$ was revealed. The pulse time arrival analysis allowed us to measure periastron passage time of the neutron star. Comparing this value with earlier published values allows to estimate orbital period and possibly its derivative, which are compatible with values obtained from RXTE-ASM data. Using INTEGRAL pointed observations we were able to obtain high quality broadband spectra of the source. The resulting spectral parameters are mostly consistent with those found in the literature. Any discrepancies between the published values and our results can be attributed to the known variability of the source and that the analyses of [2] and [3] were based on data from different instruments: RXTE and BeppoSAX, respectively. It is therefore not surprising that the fit results are somewhat different. The results of the phase resolved spectral analysis are also compatible with earlier publications and confirm that cyclotron line is indeed highly variable with phase; furthermore a correlation with the cutoff energy is observed. We were able to obtain a self-consistent description of the spectrum using a model by [17] implying that the spectrum is dominated by a thermally Comptonized bremsstrahlung emission. Even though this description is not definitive due to the strong correlations between some model parameters, it is already a highly interesting result by itself that the spectrum of GX 301-2 can be fitted this model at all, as [17] make only qualitative estimates for a limited number of sources. To further improve 
the spectral modeling with the model of [17], data of much higher quality are needed to be able to further constrain the spectral parameters.

\section{References}

[1] S. H. Pravdo and P. Ghosh, An orbital light-curve model for gx 301-2, The Astrophysical Journal 554 (Jun, 2001) 383. (c) 2001: The American Astronomical Society.

[2] I. Kreykenbohm, J. Wilms, W. Coburn, M. Kuster, R. E. Rothschild, W. A. Heindl, P. Kretschmar, and R. Staubert, The variable cyclotron line in gx 301-2, Astronomy and Astrophysics 427 (Dec, 2004) 975.

[3] A. La Barbera, A. Segreto, A. Santangelo, I. Kreykenbohm, and M. Orlandini, A study of an orbital cycle of gx 301-2 observed by bepposax, Astronomy and Astrophysics 438 (Aug, 2005) 617.

[4] N. E. White, K. O. Mason, H. E. Huckle, P. A. Charles, and P. W. Sanford, Periodic modulation of three galactic x-ray sources, Astrophysical Journal 209 (Nov, 1976) L119. A\&AA ID. AAA018.142.100.

[5] L. Kaper, A. van der Meer, and F. Najarro, Vlt/uves spectroscopy of wray 977, the hypergiant companion to the x-ray pulsar gx301-2, Astronomy and Astrophysics 457 (Oct, 2006) 595.

[6] G. E. Parkes, J. L. Culhane, K. O. Mason, and P. G. Murdin, A spectral study of wray 977, the optical counterpart of the binary x-ray pulsar 4u 1223-62, Royal Astronomical Society 191 (May, 1980) 547. A\&AA ID. AAA027.114.057.

[7] L. Kaper, H. J. G. L. M. Lamers, E. Ruymaekers, E. P. J. van den Heuvel, and E. J. Zuidervijk, Wray 977 (gx 301-2): a hypergiant with pulsar companion., Astron. Astrophys. 300 (Aug, 1995) 446.

[8] D. T. Koh, L. Bildsten, D. Chakrabarty, R. W. Nelson, T. A. Prince, B. A. Vaughan, M. H. Finger, R. B. Wilson, and B. C. Rubin, Rapid spin-up episodes in the wind-fed accreting pulsar gx 301-2, Astrophysical Journal v.479 479 (Apr, 1997) 933. (c) 1997: The American Astronomical Society.

[9] D. A. Leahy and M. Kostka, Stellar wind accretion in gx 301-2: evidence for a high-density stream, Monthly Notices of the Royal Astronomical Society 384 (Feb, 2008) 747. (c) Journal compilation (C) 2008 RAS.

[10] S. Watanabe, M. Sako, M. Ishida, Y. Ishisaki, S. M. Kahn, T. Kohmura, U. Morita, F. Nagase, F. Paerels, and T. Takahashi, Detection of a fully resolved compton shoulder of the iron $k \alpha$ line in the chandra x-ray spectrum of gx 301-2, The Astrophysical Journal 597 (Nov, 2003) L37. (c) 2003: The American Astronomical Society.

[11] P. Ubertini, F. Lebrun, G. D. Cocco, A. Bazzano, A. J. Bird, K. Broenstad, A. Goldwurm, G. L. Rosa, C. Labanti, P. Laurent, I. F. Mirabel, E. M. Quadrini, B. Ramsey, V. Reglero, L. Sabau, B. Sacco, R. Staubert, L. Vigroux, M. C. Weisskopf, and A. A. Zdziarski, Ibis: The imager on-board integral, Astronomy and Astrophysics 411 (Nov, 2003) L131.

[12] N. Lund, C. Budtz-Jørgensen, N. J. Westergaard, S. Brandt, I. L. Rasmussen, A. Hornstrup, C. A. Oxborrow, J. Chenevez, P. A. Jensen, S. Laursen, K. H. Andersen, P. B. Mogensen, I. Rasmussen, K. Omø, S. M. Pedersen, J. Polny, H. Andersson, T. Andersson, V. Kämäräinen, O. Vilhu, J. Huovelin, S. Maisala, M. Morawski, G. Juchnikowski, E. Costa, M. Feroci, A. Rubini, M. Rapisarda, E. Morelli, V. Carassiti, F. Frontera, C. Pelliciari, G. Loffredo, S. M. Núñez, V. Reglero, T. Velasco, S. Larsson, R. Svensson, A. A. Zdziarski, A. Castro-Tirado, P. Attina, M. Goria, G. Giulianelli, F. Cordero, M. Rezazad, M. Schmidt, R. Carli, C. Gomez, P. L. Jensen, 
G. Sarri, A. Tiemon, A. Orr, R. Much, P. Kretschmar, and H. W. Schnopper, Jem- $x$ : The x-ray monitor aboard integral, Astronomy and Astrophysics 411 (Nov, 2003) L231.

[13] G. Vedrenne, J.-P. Roques, V. Schönfelder, P. Mandrou, G. G. Lichti, A. von Kienlin, B. Cordier, S. Schanne, J. Knödlseder, G. Skinner, P. Jean, F. Sanchez, P. Caraveo, B. Teegarden, P. von Ballmoos, L. Bouchet, P. Paul, J. Matteson, S. Boggs, C. Wunderer, P. Leleux, G. Weidenspointner, P. Durouchoux, R. Diehl, A. Strong, M. Cassé, M. A. Clair, and Y. André, Spi: The spectrometer aboard integral, Astronomy and Astrophysics 411 (Nov, 2003) L63.

[14] N. Sato, F. Nagase, N. Kawai, R. L. Kelley, S. Rappaport, and N. E. White, Orbital elements of the binary x-ray pulsar gx 301-2, Astrophysical Journal 304 (May, 1986) 241.

[15] W. Coburn, W. A. Heindl, R. E. Rothschild, D. E. Gruber, I. Kreykenbohm, J. Wilms, P. Kretschmar, and R. Staubert, Magnetic fields of accreting x-ray pulsars with the rossi $x$-ray timing explorer, The Astrophysical Journal 580 (Nov, 2002) 394. (c) 2002: The American Astronomical Society.

[16] Y. Tanaka, Observations of compact $x$-ray sources, Radiation Hydrodynamics in Stars and Compact Objects (Jan, 1986).

[17] P. A. Becker and M. T. Wolff, Thermal and bulk comptonization in accretion-powered x-ray pulsars, The Astrophysical Journal 654 (Jan, 2007) 435. (c) 2007: The American Astronomical Society. 